\title{
Clima social familiar de mujeres consumidoras de sustancias adictivas con y sin ejercicio de la prostitución
}

\author{
Family social climate of women who consume addictive substances with \\ and without the exercise of prostitution
}

\author{
Jenny Magaly Agama Hidalgo ${ }^{1}$ \\ Fuerza Aérea del Perú (FAP) / Clínica Limatambo
}

Recibido: $26-10-18$

Aceptado: $27-12-18$

\section{Resumen}

La finalidad de este estudio es relacionar el funcionamiento familiar de consumidoras de sustancias adictivas que ejercen la prostitución con respecto a las consumidoras que no ejercen la prostitución y un grupo control que no son consumidoras ni ejercen la. prostitución. La muestra estuvo constituida por 148 mujeres que fueron divididas en tres grupos de comparación que respondieron a la escala de clima Social familiar (FES) no encontrándose diferencias significativas entre el grupo de mujeres con y sin ejercicio de la prostitución, pero si con el grupo control.

Palabras clave: Adicción; prostitución; clima social familiar.

\begin{abstract}
The purpose of this study is to relate the family functioning of consumers of addictive substances who practice prostitution with respect to consumers who do not practice prostitution and a control group who are not consumers or exercise alcohol. prostitution. The sample consisted of 148 women who were divided into three comparison groups that responded to the family social climate scale (FES), not finding significant differences between the group of women with and without exercise of prostitution, but with the control group.
\end{abstract}

Keywords: Addiction; prostitution; family functioning.

\footnotetext{
${ }^{1}$ Psicóloga en Fuerza Aérea del Perú y Clínica Limatambo. E-mail: jennyagamahidalgo@gmail.com

(C) Los autores. Este artículo es publicado por la Revista de Investigación en Psicología de la Facultad de Psicología, Universidad Nacional Mayor de San Marcos. Este es un artículo de acceso abierto, distribuido bajo los términos de la licencia Creative Commons Atribucion - No Comercia_Compartir Igual 4.0 Internacional. (http://creativecommons.org/licenses/by-nc-sa/4.0/) que permite el uso no comercial, distribución y reproducción en cualquier medio, siempre que la obra original sea debidamente citada.
} 


\section{INTRODUCCIÓN}

En la actualidad el abuso de sustancias es un problema social y legal que afecta al individuo y que va en aumento a través de los años. El consumo de sustancias psicoactivas, no solo repercute en la persona, en su salud física, sino también lo psicológico, social y espiritual y trae consigo graves implicancias en el desarrollo moral económico de la sociedad y por ende del país.

Es así, que en un estudio de [CEDRO] en el año 2015 denominado "Epidemiologia de Drogas de la población Urbana Peruana 2015", indica que actualmente hay más de un millón de personas en nuestro país que han consumido alguna droga ilegal. Sumado a ello, nuestro país es considerado como el primer productor de drogas cocainitas a nivel mundial (DEVIDA,2015). Organismos internacionales como la [OMS] (2014) ha llegado a considerar el consumo como un problema de salud pública

La problemática de las drogas es compleja y necesita ser estudiado desde una perspectiva multidisciplinaria requiere que se tomen en cuenta las áreas biológicas, sociales, culturales y ambientales que posibiliten comprender como interactúan los microsistemas del individuo y la familia. Ya que la familia es un factor de gran importancia para poder hallar la etiología y el tratamiento (Bronfenbrenner 2005, Pons, Buelga 2011)

Se sabe que la disfuncionalidad familiar es uno de los factores de riesgo, familias permisivas con poco control, poca supervisión, escasa comunicación (Abar, 2012) La familia que recibe las consecuencias directas como la agresividad y la obsesión por querer controlar la conducta del adicto, generándose así un círculo vicioso de sufrimientos compartidos. Así mismo, repercute significativamente en la aparición de problemas de índole social como la delincuencia, prostitución, mortalidad

Para el presente estudio abordaremos la problemática de prostitución en mujeres consumidoras de sustancia psicoactivas comparándolo con un grupo control.

La relación que existe ente el consumo de drogas y la prostitución parece sobrentendida, no obstante, existen pocos estudios relacionados al consumo de la sustancia psicoactiva y el ejercicio de la prostitución Alvares (2016)

Pese a que el consumo es una dificultad por la que atraviesa la sociedad y afecta la salud que tiene un gran impacto en las mujeres, ya que las llamadas trabajadoras sexuales por un tema de género son vistas con poca importancia, de la misma manera sus derechos, sumado a su situación de vida y su salud

Existe un estudio del consumo de sustancias psicoactivas en mujeres que ejercían la prostitución en México en la ciudad de La Merced. Rodríguez, 
Gutiérrez, y Vega, (2003). Esta ciudad se conoce por tremendos índices de problemática psicosocial, tales como pobreza, violencia y marginalidad. En esta situación las mujeres presentan mayor vulnerabilidad, lo que muchas veces coincide con la acción de explotadores. Se Utilizó el método cualitativo y una sucesión de entrevistas profundas en 14 mujeres. Las entrevistas fueron grabadas $\mathrm{y}$ en promedio se realizaban en hora y media. Los hallazgos muestran gran vinculación entre el ambiente circundante a la prostitución y la dependencia a sustancias psicoactivas. Las participantes en su mayoría indicaron que consumían drogas ilegales y alcohol.

El consumo problemático de drogas es un problema de salud pública muy complejo, se requiere políticas públicas para controlar esta conducta patológica, asimismo; que se aborde desde un punto de vista explicativo y no solo descriptivo la problemática de las drogas; así como de los estudios del factor ambiental que nos lleve a comprender las interacciones de los microsistemas del individuo y la familia (Bronfenbrenner, 2015; Pons \& Buelga, 2011). En este estudio se toma en cuenta los componentes multifactoriales, se pone énfasis en los factores familiares y es relevante tener un abordaje que incluya a la familia debido a que es relevante pues es el primer medio de sociabilización de las personas.

Relacionado al consumo de la droga, los estudios señalan algunos aspectos de riesgo, tales como: vivir con familias no funcionales, violencia familiar, poco asesoramiento en estilos de crianza, escasa comunicación -con la familia o ver un ambiente familiar negativo, carencia de afecto y aceptación (Buelga \& Pons, 2004), patrones de crianza condescendientes, estilos familiares que aumentan la posibilidad de ser impulsivos (Patock-Peckham y Morgan-López, 2006), padres con antecedentes de alcoholismo, escaso control y mala relación con sus hijos (Abar, 2012).

Por otro lado, Ortiz, Soriano, Meza, Martínez y Galván (2006) investigaron la drogadicción en una muestra de 694 personas, en su mayoría hombres (618), con edades entre los 11 y los 30 años o más, que pertenecían a organismos de salud e instituciones encargados del área de justicia. Hallaron que en hombres y mujeres el principal factor del consumo de sustancia psicoactiva son los problemas con la familia.

De la misma manera, entre las personas que piden tratamiento para las dependencias a sustancias psicoactivas se aprecia que ser parte de familias disfuncionales presentan mayor sensibilidad para consumir drogas, ya que se los problemas en la comunicación y emocionales están muy asociados a esta problemática (Iraurgi, Sanz y Martínez, 2014).

Teniendo en cuenta los precedentes, podríamos considerar hipotéticamente que las consumidoras de drogas presentarían mayores disturbios en el funcionamiento 
familiar en comparación con el grupo que no se droga ni ejerce la prostitución; y asimismo consideramos que las consumidoras que se prostituyen presentarían mayor disfuncionalidad familiar en relación a aquellas que solo consumen drogas.

\section{Formulación del problema}

¿Las consumidoras de sustancias adictivas que ejercen la prostitución y los que no ejercen la prostitución tendrían mayor problemática en su funcionamiento familiar que aquellos participantes sin drogadicción y sin ejercicio de la prostitución?

\section{Objetivos}

- Describir la dinámica de las mujeres dependientes a sustancias psicoactivas sin ejercicio de la prostitución

- Describir la dinámica de las mujeres dependientes a sustancias psicoactivas con ejercicio de la prostitución

- Describir la dinámica de las mujeres no dependientes a sustancias psicoactivas sin ejercicio de la prostitución

\section{Método}

\section{Tipo y Diseño de investigación}

La siguiente investigación tiene un diseño de corte transversal, es no experimental y específicamente con un modelo de tres muestras independientes. Hernández, Fernández y Baptista (2014)

\section{Muestra}

Se utilizó una muestra no probabilística de personas voluntarias. Los participantes fueron un grupo de $(\mathrm{n}=50)$ y dos grupos de $(\mathrm{n}=49)$ se concretó en acuerdo con la organización que permitió acceder a su población hasta poder terminar con la cantidad determinada. Fueron evaluadas 148 mujeres con edades entre 18 y 35 años, que se organizaron en 3 en función al consumo de drogas y el ejercicio de la prostitución

Grupo 1, Consumidores recurrentes con ejercicio de la prostitución: conformado por 49 consumidoras de sustancias adictivas que ejercen la prostitución, cuyo consumo de sustancias psicoactivas eran tanto legales como ilegales, cuyos criterios eran que consumían más de dos días por semana y reportaba dificultad en el ámbito social familiar y escolar motivo por el cual estaban en tratamiento de rehabilitación residencial

Grupo 2, Consumidoras recurrentes sin ejercicio de la prostitución: integrado por 49 cuyo consumo de sustancia psicoactiva era legales e ilegales cuyos criterios 
eran que consumían más de dos días por semana y reportaba dificultad en el ámbito social familiar y escolar motivo por el cual estaban en tratamiento residencial. Toman más de dos copas por ocasión, con una frecuencia semanal mayor o igual a 2 días, presentando reportes de problemas en la familia, lo académico y/o lo social, siendo por ello internados en este centro de para su rehabilitación.

Grupo 3, No ingieren sustancias psicoactivas y no ejercen la prostitución: conformado por 50 personas. Este grupo refiere solo haber consumido una vez alcohol o no haber consumido nunca drogas.

\section{Técnicas e instrumentos de recolección de datos}

Serealizaron coordinaciones previas con las comunidades terapéuticas, de las cuales se obtuvo el permiso respectivo para la para la aplicación de los instrumentos en fechas programadas. Los evaluados recibieron el consentimiento informado, con las indicaciones universales éticas de confidencialidad, anonimato, voluntariedad, etc. Los instrumentos usados fueron:

a. Cuestionario descriptivo: contiene los datos demográficos, edad, sexo, grado de instrucción y tiempo de internamiento y condición.

b. Escala de Clima Social en la Familia (FES), (Moos, Moos \& Trickett, 1980).

Nombre original: Escala del clima Social en la Familia (FES).

Adaptación: Española Estudios TEA.

Confiabilidad: Los estudios en Lima han trabajado con la consistencia interna obteniendo coeficientes que oscilan entre 0.88 a 0.91 , siendo la media igual a 0.89, Ayala, Fulgencio, Chaparro y Pedroza, 2000.

Administración: Individual y colectiva.

Significación: Es una escala individual cuyo propósito es el examen de las características sociales y del ambiente en la familia.

Áreas que mide:

1. Dimensión de Relaciones: es la que evalúa el grado de comunicación, expresión e interacción de la familia (Cohesión, expresividad y conflicto).

2. Dimensión de desarrollo: Que evalúa la importancia de la familia de procesos de desarrollo personal que pueden ser fomentado por la vida o no por la vida en común, está compuesto por (Autonomía, Actuación, Intelectual Cultural, Social Recreativo y moralidad -Religiosa). 
3. Estabilidad: Que proporciona información sobre la estructura y organización de la familia y sobre el grado de control que normalmente ejercen unos miembros de la familia sobre otros. Está compuesto por dos áreas (Organización y Confiabilidad)

En Perú se obtuvo, la confiabilidad estudiada a través del alfa total de Cronbach fue de 0.78 y con respecto a la validez de constructo, el análisis factorial encontró los mismos factores que en la prueba original (Ayala, Fulgencio, Chaparro y Pedroza, 2000).

Tabla 1

Características de los participantes

\begin{tabular}{|c|c|c|c|c|}
\hline Grupo & & $\begin{array}{l}\text { Consumidoras } \\
\text { que ejercieron } \\
\text { prostitución }\end{array}$ & $\begin{array}{l}\text { Consumidoras } \\
\text { que no ejercieron } \\
\text { prostitución }\end{array}$ & $\begin{array}{l}\text { No consumidoras } \\
\text { que no ejercieron } \\
\text { prostitución }\end{array}$ \\
\hline \multirow[t]{3}{*}{ Edad } & De 18 a 20 años & $26.5 \%$ & $22.0 \%$ & $40.8 \%$ \\
\hline & De 21 a 30 años & $65.3 \%$ & $66.0 \%$ & $59.2 \%$ \\
\hline & De 31 a 38 años & $8.2 \%$ & $12.0 \%$ & $0.0 \%$ \\
\hline \multirow{3}{*}{$\begin{array}{l}\text { Grado de } \\
\text { instrucción }\end{array}$} & Secundaria incompleta & $34.7 \%$ & $36.0 \%$ & $0.0 \%$ \\
\hline & Secundaria completa & $49.0 \%$ & $48.0 \%$ & $0.0 \%$ \\
\hline & Superior incompleta & $16.3 \%$ & $16.0 \%$ & $100 \%$ \\
\hline \multirow{3}{*}{ Estado civil } & Soltera & $83.7 \%$ & $80.0 \%$ & $100.0 \%$ \\
\hline & Casada & $10.2 \%$ & $20.0 \%$ & $0.0 \%$ \\
\hline & Conviviente & $6.1 \%$ & $0.0 \%$ & $0.0 \%$ \\
\hline \multirow{3}{*}{$\begin{array}{l}\text { Tiempo de } \\
\text { internamiento }\end{array}$} & De 1 un a 1 año & $26.5 \%$ & $24.0 \%$ & $0.0 \%$ \\
\hline & De 13 meses a 2 años & $65.3 \%$ & $68.0 \%$ & $0.0 \%$ \\
\hline & Más de 2 años & $8.2 \%$ & $8.0 \%$ & $0.0 \%$ \\
\hline
\end{tabular}

\section{Procedimiento}

El estudio se realizó en una organización de tratamiento de rehabilitación residencial de Lima en donde se encontraban internas las consumidoras de drogas. Asimismo se consideraron instituciones educativas para el grupo control.

Se inició presentando el proyecto a los responsables de los órganos participantes, cumpliendo los criterios éticos necesarios en toda investigación con personas, respetándose la privacidad personal y el derecho a retirarse si así lo desearan. Los responsables de cada organización firmaron el consentimiento informado. 


\section{Análisis de datos}

Se realizaron estudios exploratorios y descriptivos, para posteriormente someter estos datos al Análisis de Varianza Unidireccional (ANOVA de una via), para poder comparar las subescalas en cada grupo y describir su funcionamiento familiar. Como prueba de comprobación Pos-Hoc se ha utilizado Tukey.

\section{Resultados}

Al inicio se examinó la dinámica familiar de los tres grupos, mediante matrices que jerarquizan las subescalas en cada grupo a través de la media, para poder visualizar la percepción que tiene cada grupo respecto al funcionamiento familiar. Es así que las subescala con media mayor es la que ocupa el primer lugar y así en descendiente, tal como se observa en la tabla 2.

En el caso de las consumidoras que han ejercido la prostitución, ellas otorgan mayor importancia entre las dimensiones del funcionamiento familiar al componente religioso conflicto y autonomía. En un lugar intermedio se encuentran los aspectos social-recreativo, expresión, control, actuación y organización. De otro lado, la cohesión y los aspectos intelectual-culturales se ubican en los últimos lugares.

En este segundo grupo de adictas que no han ejercido la prostitución las integrantes perciben en los miembros de sus familias mayor orientación hacia aspecto religiosos pero acompañados de constantes conflictos y una mayor búsqueda de autonomía en la consecución de sus propios intereses. Asimismo, Además la consideran como un entorno con poca unión familiar y que presta menor atención a actividades intelectuales y culturales.

En el caso de las consumidoras que no ejercieron la prostitución, se encuentran coincidencias con las consumidoras que sí la ejercieron. En ese sentido, también perciben como características de mayor importancia en su familia al aspecto moral religioso, la presencia de conflicto y la tendencia en sus miembros a la consecución de sus propios intereses, además de otorgar poca presencia a la unión familiar y a las actividades intelectuales y culturales.

De otro lado, el grupo de control (mujeres que no consumen y que no han ejercido la prostitución) se diferencia de los grupos de consumidoras. En este caso perciben en sus familias mayor presencia de organización, seguido de moral religioso e intelectual cultural. En un lugar intermedio se encuentran cohesión, actuación, social-recreativo, autonomía y expresión. Y en los últimos lugares están control de conflicto. De lo anterior, se puede inferir que en el caso del grupo de control, perciben en sus familias mayor organización en las actividades que realizan, mayor tendencia hacia aspectos morales y religiosos y que se otorgan 
importancia a las actividades intelectuales y culturales, así como menor presencia de control y conflicto.

Tabla 2

Matrices de funcionamiento familiar de los participantes

\begin{tabular}{|c|c|c|c|c|c|c|c|c|c|}
\hline \multirow{2}{*}{ Jerarquía } & \multicolumn{3}{|c|}{$\begin{array}{l}\text { Consumidoras quienes } \\
\text { ejercieron prostitución }\end{array}$} & \multicolumn{3}{|c|}{$\begin{array}{c}\text { Consumidoras quienes no } \\
\text { ejercieron prostitución }\end{array}$} & \multicolumn{3}{|c|}{$\begin{array}{l}\text { No consumidoras que no } \\
\text { ejercieron prostitución }\end{array}$} \\
\hline & Subescala & Media & DE & Subescala & Media & DE & Subescala & Media & DE \\
\hline $1^{\circ}$ & $\begin{array}{c}\text { Moral } \\
\text { Religioso }\end{array}$ & 6.39 & .671 & $\begin{array}{c}\text { Moral } \\
\text { Religioso }\end{array}$ & 6.24 & .771 & Organización & 8.39 & .786 \\
\hline $2^{\circ}$ & Conflicto & 5.55 & 1.815 & Conflicto & 5.58 & 1.785 & Moral Religioso & 7.22 & .963 \\
\hline $3^{\circ}$ & Autonomía & 5.18 & 1.074 & Autonomía & 5.14 & 1.088 & $\begin{array}{l}\text { Intelectual } \\
\text { Cultural }\end{array}$ & 7.04 & .455 \\
\hline $4^{\circ}$ & $\begin{array}{c}\text { Social } \\
\text { Recreativo }\end{array}$ & 4.02 & .924 & $\begin{array}{c}\text { Social } \\
\text { Recreativo }\end{array}$ & 3.92 & .986 & Cohesión & 6.73 & 1.056 \\
\hline $5^{\circ}$ & Expresión & 3.82 & 1.074 & Control & 3.90 & 1.035 & Actuación & 6.55 & .792 \\
\hline $6^{\circ}$ & Control & 3.69 & 1.045 & Expresión & 3.90 & 1.093 & $\begin{array}{c}\text { Social } \\
\text { Recreativo }\end{array}$ & 6.29 & .764 \\
\hline $7^{\circ}$ & Actuación & 3.35 & 2.016 & Actuación & 3.34 & 2.016 & Autonomía & 5.90 & .918 \\
\hline $8^{\circ}$ & Organización & 2.88 & 1.033 & Organización & 2.90 & 1.015 & Expresión & 5.78 & .743 \\
\hline $9^{\circ}$ & Cohesión & 2.57 & .866 & Cohesión & 2.64 & .875 & Control & 5.16 & .717 \\
\hline $10^{\circ}$ & $\begin{array}{c}\text { Intelectual } \\
\text { Cultural }\end{array}$ & 2.08 & .862 & $\begin{array}{c}\text { Intelectual } \\
\text { Cultural }\end{array}$ & 2.18 & .962 & Conflicto & 2.02 & .924 \\
\hline
\end{tabular}

En un primer acercamiento a nivel descriptivo se observa que los grupos de consumidoras mantienen coincidencias en las subdimensiones que perciben de mayor importancia en sus familias, en ese sentido estos grupos se diferencian del grupo control. Cabe resaltar que las dimensiones que mayor diferencia presentan entre los grupos de consumidoras y no consumidoras son conflicto y actividades intelectual-culturales.

Luego del análisis descriptivo fue menester corroborar si existen diferencias estadísticamente significativas entre los tres grupos en cuanto a las subescalas para lo cual se utilizó la prueba ANOVA de una vía. De acuerdo a esta prueba se encuentra en los grupos diferencias entre los tres grupos en todas las subescalas. Estos resultados se muestran en la tabla 3.

Especificando lo anterior, en el caso de las subescalas de cohesión, expresión, autonomía, actuación, intelectual-cultural, social-recreativo, moral-religioso, organización y control los grupos de consumidoras presentan niveles semejantes entre sí, mientras que ambos se diferencian del grupo de control, la cual presenta una media significativamente mayor a las medias de los grupos de consumidoras. De otro lado, en el caso de la subescala de conflicto los grupos de consumidoras 
presentan medias similares, pero que son a su vez significativamente mayores a la media del grupo control.

Tabla 3

Comparación del funcionamiento familiar entre grupos

\begin{tabular}{lccccccc}
\hline $\begin{array}{c}\text { Funcionamiento } \\
\text { familiar }\end{array}$ & \multicolumn{2}{c}{$\begin{array}{c}\text { Consumidoras } \\
\text { que ejercieron } \\
\text { prostitución }\end{array}$} & \multicolumn{2}{c}{$\begin{array}{c}\text { Consumidoras } \\
\text { que no ejercieron } \\
\text { prostitución }\end{array}$} & $\begin{array}{c}\text { No consumidoras } \\
\text { que no ejercieron } \\
\text { prostitución }\end{array}$ & ANOVA \\
\hline Subescalas & Media & DE & Media & DE & Media & DE & F \\
\hline Cohesión & 2.57 & .866 & 2.64 & .875 & 6.73 & 1.056 & $318.834^{* *}$ \\
Expresión & 3.82 & 1.074 & 3.90 & 1.035 & 5.78 & .743 & $65.075^{* *}$ \\
Conflicto & 5.55 & 1.815 & 5.58 & 1.785 & 2.02 & .924 & $84.072^{* *}$ \\
Autonomía & 5.18 & 1.074 & 5.14 & 1.088 & 5.90 & .918 & $8.397^{* *}$ \\
Actuación & 3.35 & 2.016 & 3.34 & 2.016 & 6.55 & .792 & $57.603^{* *}$ \\
Intelectual & 2.08 & .862 & 2.18 & .962 & 7.04 & .455 & $629.807^{* *}$ \\
Cultural & 4.02 & .924 & 3.92 & .986 & 6.29 & .764 & $109.417^{* *}$ \\
Social Recreativo & 6.39 & .671 & 6.24 & .771 & 7.22 & .963 & $21.125^{* *}$ \\
Moral Religioso & 2.88 & 1.033 & 2.90 & 1.015 & 8.39 & .786 & $546.853^{* *}$ \\
Organización & 3.69 & 1.045 & 3.90 & 1.093 & 5.16 & .717 & $33.228^{* *}$ \\
Control & & & & & &
\end{tabular}

Nota: $* * p<0.001$

\section{Discusión}

En el ámbito descriptivo en las consumidoras que no ejercieron la prostitución, se encuentran coincidencias con las consumidoras que las ejercieron. En este sentido también perciben como característica de mayor importancia, la presencia de conflicto y la tendencia en sus miembros a la consecución de sus propios intereses, así como de otorgar poca presencia a la unión familiar. Pese a ello también puntúan alto en el área religioso.

Por otro lado existen diferencias con el grupo control, es decir las mujeres que no consumen y que no han ejercido la prostitución dan mayor importancia a la organización, lo moral, religioso e intelectual cultural.

El análisis de los resultados nos proporciona información importante, es así que (Beck 1999) menciona que las adictas que han ejercido la prostitución y las que no han ejercido la prostitución otorgan mayor importancia a la dimensión de Religión, debido a que por lo general en el caso de las consumidoras de sustancias psicoactivas presentan un sentimiento de culpa incrementado, debido a que tienden a considerar que la sociedad las percibe como inmorales llegando a considerarse a ellas mismas como personas indignas por ser adictas. 
En lo concerniente a la dimensión Autonomía también ambos grupos de consumidoras puntúan alto, ello concuerda con uno de los cuatro procesos cognitivo que incluye Marlatt (1985), Autoeficacia, es decir, el adicto tiene la idea que ni él ni nadie lo hará cambiar, y no necesita de nadie, que puede hacerlo todo solo, incluso que puede dejar el consumo cuando él quiera, tendiendo a presentar una conducta de autosuficiencia. Cabe señalar que también ambos grupos también puntúan alto en la dimensión de conflicto, pues cuando la enfermedad se cronifica, el adicto llega a tener conflictos en el trabajo, con la familia, la pareja, amistades, etc. (DSM-V).

En lo concerniente al ámbito cultural - intelectual las adictas con y sin ejercicio de la prostitución puntúan bajo esto se relaciona con los estudios de prostitución de Díaz y González, 2007, quienes mencionan que hay todo un conjunto de factores que inciden en el surgimiento de la prostitución, entre ellos los problemas con la transmisión de valores y medidas económicas imprescindibles.

En el ámbito familiar las adictas con y sin ejercicio de la prostitución tienen mayores problemas con la familia en comparación con el grupo control. Asimismo, se observó que las adictas que no ejercen la prostitución presentan ligeramente un mejor funcionamiento familiar en comparación con las adictas que ejercen la prostitución.

En lo que concierne a cohesión las consumidoras prestan poca importancia a la unión familiar, esto concuerda con los estudios de (Sáenz y Medici, 2010) quienes refieren que los adictos en tratamiento evalúan negativamente la relación afectiva y vínculo que han mantenido durante la infancia y adolescencia con la familia. Asimismo, (Ortiz, Meza, Martínez, Galván 2016) encontraron que el primer antecedente del problema de drogas son los problemas con la familia. Sumado a ello (Abar, 2012) pone énfasis en padres de perfiles pro-alcohólicos con patrones de crianza permisivos y poco control.

\section{CONCLUSIONES}

1. A nivel descriptivo se observa que los grupos de consumidoras mantienen coincidencias en que perciben mayor importancia en sus familias, en ese sentido estos grupos se diferencian del grupo control. Cabe resaltar que las dimensiones que mayor diferencia presentan entre los grupos de consumidoras y no consumidoras son conflicto y actividades intelectualculturales.

2. Los tres grupos presentan involucramiento religioso, pero en las de adictas que han ejercido la prostitución y las que no han ejercido la prostitución otorgan mayor importancia a la dimensión de Religión, debido a que por lo general presentan un sentimiento de culpa incrementado y no se 
perdonan a sí mismas, debido a que tienden a considerar que la sociedad las percibe como inmorales llegando a considerarse a ellas mismas como personas indignas por ser adictas.

3. A nivel general se encuentra diferencias entre el grupo de adictas con o sin ejercicio de la prostitución y las del grupo control.

4. Los tres grupos presentan involucramiento religioso, siendo inclusive ligeramente más alto el nivel religioso en las adictas que ejercen la prostitución, pues tienden a presentar sentimiento de culpa mayores y a no perdonarse a sí mismas por lo que buscan soporte espiritual y mayor involucramiento religioso.

5. En el ámbito familiar las adictas con y sin ejercicio de la prostitución tienen mayores problemas con la familia en comparación con el grupo control. Asimismo, se observó que las adictas que no ejercen la prostitución presentan ligeramente un mejor funcionamiento familiar en comparación con las adictas que ejercen la prostitución.

6. En lo que concierne a cohesión las adictas prestan poca importancia a la unión familiar, los adictos en tratamiento evalúan negativamente la relación afectiva y vínculo que han sostenido durante la infancia y adolescencia con la familia. Asimismo, se encontró que el primer antecedente del problema de drogas son los problemas con la familia. Sumado a ello están los padres de con disposición genética al alcoholismo con patrones de crianza permisivos y poco control.

7. Se ha comprobado la existencia de diferencias significantes del clima social familiar al comparar a mujeres consumidoras de Sustancias Psicoactivas con y sin ejercicio de la prostitución y el grupo control (las mujeres que no consumen ni ejercen la prostitución).

\section{REFERENCIAS}

Abar, C. (2012). Examining the relationship between parenting types and patterns of student alcohol-related behavior during the transition to college. Psychology of Addictive Behaviors, 26(1), 20-29.

Alvares, L (2016) Adicción y ejercicio de la prostitution Revista de psicologia de la Universidad de Malaga.

Ayala, H., Fulgencio, M., Chaparro, A. y Pedroza, F. (2000). Resultados preliminares del proyecto estudio longitudinal del desarrollo de la conducta agresiva en niños y su relación con el establecimiento de conducta antisocial en la adolescencia. Revista Mexicana Análisis de la Conducta, 26, 65-89. 
Beck, A., Wright, F., Newman, C y Liese, B. (1999). Terapia cognitiva de la drogodependencia. Barcelona: PaidósIbérica.

Bronfenbrenner, U. (2015). Making human beings human: Bioecological perspectives on human development. Thousand Oaks: Sage.

Buelga, S. y Pons, J. (2004). Alcohol y Adolescencia: ¿Cuál es el papel de la familia?. En L. Gómez (coord.). Encuentros en Psicología Social. Málaga: Aljibe.

Centro de información y educación para la prevención del abuso de drogas (CEDRO) (2015). El problema de las drogas en el Perú 2015 Lima : CEDRO.

Comisión Nacional para el desarrollo y vida sin drogas (DEVIDA) (2015) Agenda Nacional de Investigación para la lucha contra las drogas. Lima: DEVIDA

Díaz, T. y Gonzalez, G (2007). Cultura y prostitución: Una posible solución. Revista de sociología Vol 52.

Hernández, R.; Fernández, C, y Batista , P. (2014). Metodología de la Investigación 6ta edición ed México DF: Mc Gray Hill

Iraurgi, I., Sanz, M. y Martínez, A. (2014). Funcionamiento familiar y severidad de los problemas asociados a la adicción a drogas en personas que solicitan tratamiento. Adicciones, 16(3), 185-195.

Marlatt, G (1993). La prevención de recaídas en las conductas adictivas. Barcelona: Neurociencias

Organización mundial de la salud (2014). Informe mundial sobre alcohol y salud 2014. Luxenburgo: OMS

Ortiz, A., Soriano, A., Meza, D., Martínez, R. y Galván, J. (2006). Uso de sustancias entre hombres y mujeres, semejanzas y diferencias. Resultados del sistema se reporte de información de drogas. Salud Mental, 29(5), 32-37.

Patock-Peckham, J. A. \& Morgan-López, A.A. (2006). College drinking behaviors: mediational links between parenting styles, impulse control, and alcohol-related outcomes. Psycholgy of Addictive Behaviors. Jun;20(2):117-25.

Pons, J. y Buelga, S. (2011). Factores asociados al consumo juvenil de alcohol: una revisión desde una perspectiva psicosocial y ecológica. Psychosocial Intervention, 20(1), 75-94.

Rodríguez, E., Gutiérrez, R y Vega, L. (2003). Consumo de drogas en mujeres dedicadas a la prostitución: la zona de La Merced. Salud Mental, Vol. 26, №. 5, págs. 73-81.

Sáenz, I. y Medici, S. (2010). La relación afectiva y vincular de los adictos con la familia en la infancia y la adolescencia. Tesis Licenciatura en Psicología, Universidad Abierta Interamericana. 